\title{
Experimental Testing of Least-Square Procedure for Determining Railgun Plasma Current Distribution
}

\author{
KENNETH K. COBB
}

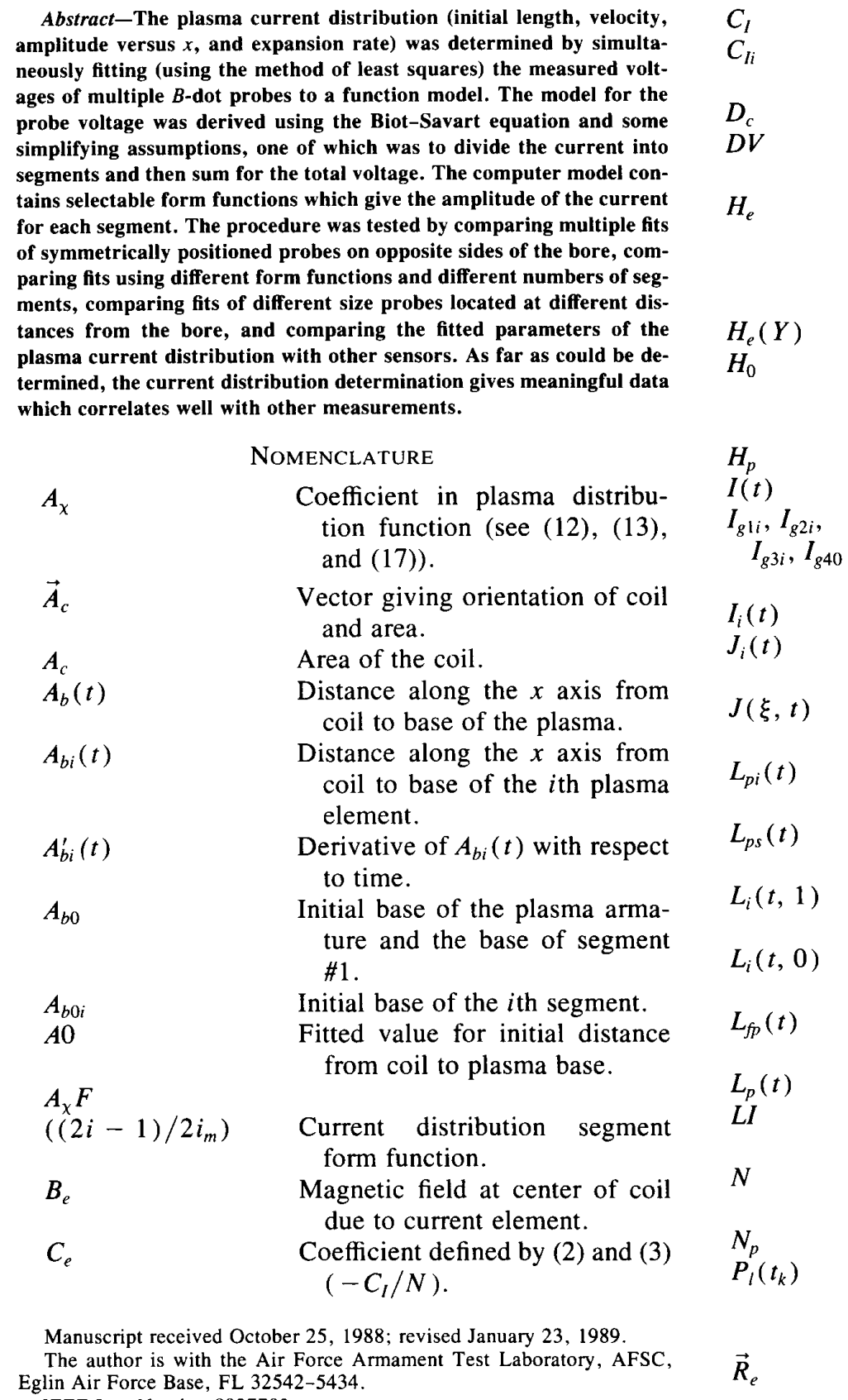

Voltage function coefficient (7).

Voltage function coefficient (7)

$\left(C_{I}\right) *$ (form function).

Diameter of coil.

Fitted value for the expansion rate of plasma.

$y$ distance from the current element to the plane parallel to the $X-Z$ plane containing the $\dot{B}$ coil.

$H_{0}-Y$.

$Y$ distance from the $X-Z$ plane to a plane parallel to the $X-Z$ plane containing the $\dot{B}$ coil.

Width of the plasma armature.

Total plasma current.

Integrals of the $i$ th segment of voltage function.

Current in the $i$ th segment.

Current density of the $i$ th segment.

Current density in plasma (see (3)).

Length of the $i$ th plasma segment.

Length of the plasma segment for given model.

Defined by (24) (see voltage function).

Defined by (27) (see voltage function).

Distance from the front face of plasma to the coil probe.

Total length of plasma.

Fitted value for the initial length of plasma.

Number of data points in leastsquare fit.

Number of parameters being fit.

Partial of voltage function with respect to $l$ parameter evaluated at time $t_{k}$.

Vector position of the coil center in plane parallel to rails mid- 
way between rails from midpoint of current element.

$R_{e}(\xi, Y, t)$

$R_{p}(\xi, t)$

$R_{0}(\xi, t)$

$R_{p i}(t, 1), R_{0 i}(t, 1)$,

$R_{p i}(t, 0), R_{0 i}(t, 0)$

$V_{k}$

$V P$

$\vec{W}$

$W$

$X, Y, Z$

$\bar{X}$

$X_{i}$

$Z_{i}$

$a_{i}$

$a_{p}$

$f(t)$

$f_{i}$

$f_{i}(t)$

$i_{m}$

$p_{i}$

$t$

$t_{k}$

$i$

$k$

$l, m$

$\beta$

$\gamma$

$\delta a_{i}$

$\delta V_{p}$

$\mu_{0}$

$\xi$

$\sigma$

$\phi_{e}$

$\chi_{i}$
Magnitude of the vector $\vec{R}_{e}$ given in terms of $\xi, Y$, and $t$ (see (1) and (3)).

Identical to $R_{e}\left(\xi, H_{p}, t\right)$ (see (3)).

Identical to $R_{e}(\xi, 0, t)$ (see (3)).

See voltage function.

Measured voltage at time $t_{k}$.

Fitted value for the initial plasma velocity.

Vector current element distance between rail.

Perpendicular distance between rails.

Coordinates (see Fig. 1).

Gaussian mean (see (38) through (41)).

$X$ distance from base of the $i$ th segment.

In function (see (18)).

Amplitude of the segment in amplitude form function.

Projectile acceleration.

Abbreviation for $f(t)$.

$\dot{B}$ coil voltage function.

Abbreviation for $f_{i}(t)$.

$\dot{B}$ coil voltage function for the $i$ th segment.

Number of segments.

$i$ th parameter in the least-square fit.

Time.

$k$ th data time point.

Subscript indicating segment number.

Subscript indicating data point number.

Subscript indicating $l, m$ parameter.

Slope of current function (see (36)).

Plasma acceleration rate in excess of the projectile.

Difference between acceleration of the $i$ segment base and plasma front.

Expansion velocity of plasma.

Permeability of free space.

Nondimensional length of the plasma segment.

Gaussian variance (see (38) through (41)).

Coil flux caused by the armature current element.

Variable in function (see (18)).

\section{INTRODUCTION}

THE plasma armature is a key element which limits the efficiency and performance of an electromagnetic railgun. To understand the plasma armature, it is essential to measure its current distribution. This has been done by using small induction coils [1], [2]. The current distribution along with the railgun current and muzzle voltage may be used in conjunction with the equilibrium model of Powell and Batteh [3] to determine all aspects of the plasma, including the mass [4].

In [1], a procedure was derived to determine the current distribution in the plasma armature from the measured voltage on a small induction coil oriented to sense only the armature current. A model for the voltage was derived by integrating the coil magnetic-field contributions (using the Biot-Savart equation for the field due to a single current element) over the current elements contained in the armature model. The time rate of change of the field flux was taken to give the coil voltage.

The parameters in the model allow the plasma to expand or contract with acceleration, to have varying total current, and to have an arbitrary functional geometrical distribution of current along the axis of the launcher. The closed-form solution requires the assumption that the current in the plasma is made up of sheets with the flow being perpendicular to the rails and the current amplitude being a function of time and the distance from the projectile base. The closed-form solution also requires the current distribution to be segmented in the bore direction with the current amplitude in any segment given by a form function.

The important feature of the closed-form solution is that it allows the use of the method of least squares in analyzing the data. The least-square algorithm allows for fitting (solving for) the parameters of the model which give the current distribution. Comparing the model calculations with other sensors gives a good check on the validity of the model. The model allows the substitution of various functional forms describing the geometrical distribution of current in the axis direction.

In this paper, this procedure for the determination of the current distribution was improved and expanded, and the approach was evaluated using test data. Improvement in the method included: 1) Simplification of the derived voltage function, 2) adding the capability to reduce data from multiple probes simultaneously, and 3) adding an additional form function which gives an alternate description of the geometrical distribution of the current.

A test was designed which had symmetrically placed coils on both sides of the bore. The data from this test was used to compare: i) The multiple fits of probes on one side of the bore with the multiple fits of symmetrically located probes on the other side of the bore, ii) the multiple fits using different form functions and different numbers of segments, and iii) the fits of different size probes located at different distances from the bore. Other test data were used to compare fitted parameters with data from other sensors. 


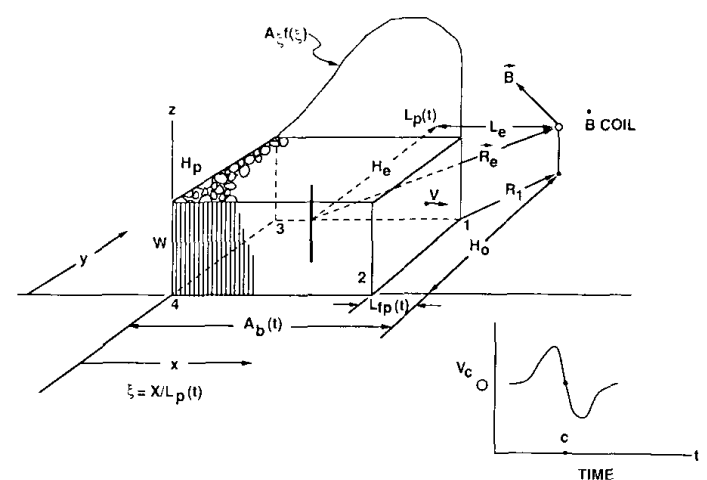

Fig. 1. Pictorial of plasma armature.

\section{The Voltage Function}

The voltage function was derived using the Biot-Savart equation as was described in [1]. The geometry for this derivation is shown in Fig. 1, where the small coil is located midway between the rails and is oriented to pickup rail current only. As was shown, the magnetic field for an element of current is given by

$$
\begin{aligned}
\vec{B}_{e} & =\left(\mu_{0} I_{e} \vec{W} \times \vec{R}_{e}\right) / 4 \pi R_{e}^{3}\left(1+W^{2} / 4 R_{e}^{2}\right)^{1 / 2} \\
& \simeq\left(\mu_{0} I_{e} \vec{W} \times \vec{R}_{e}\right) / 4 \pi R_{e}^{3} .
\end{aligned}
$$

The approximate form of (1) was used in the derivation of the voltage function, and is less accurate as the probe is placed closer to the bore (this approximation will be discussed as it effects some of the test data). The flux linking the coil due to a current element is

$$
\phi_{e}=\vec{B}_{e} \cdot \vec{A}_{c}=\mu_{0} I_{e} W A_{c} H_{e} / 4 \pi R_{e}^{3} .
$$

This may be integrated over the transverse direction (sheets of current transverse to the bore-assumed constant) to give the total flux as

$$
\begin{aligned}
\phi_{c}(t)= & C_{e} \int_{0}^{1} J(\xi, t)\left[\left(1 / R_{p}(\xi, t)\right)\right. \\
& \left.-\left(1 / R_{0}(\xi, t)\right)\right] L_{p}(t) d \xi
\end{aligned}
$$

where $R_{p}(\xi, t) \equiv R_{e}\left(\xi, H_{p}, t\right), R_{0}(\xi, t) \equiv R_{e}(\xi, 0, t)$, and the variables in (2) were taken as

$$
\begin{aligned}
R_{e}(\xi, Y, t) & =\left[\left(A_{b}(t)-\xi L_{p}(t)\right)^{2}+\left(H_{0}-Y\right)^{2}\right]^{1 / 2} \\
H_{e}(Y) & =H_{0}-Y \\
\xi & \equiv X / L_{p}(t) \\
I_{e} & =J(\xi, t) d Y d \xi L_{p}(t)
\end{aligned}
$$

where $A_{b}(t)=$ the distance from the base of the plasma to the coil along the bore, and $L_{p}(t)=$ the total length of the plasma.

Equation (3) is integrable in closed form if $J(\xi, t)$ is constant with respect to $\xi$. In order to get a closed-form integral and still simulate a variable current distribution in the bore $(X)$ direction, the armature current is divided into segments with the current density constant over the

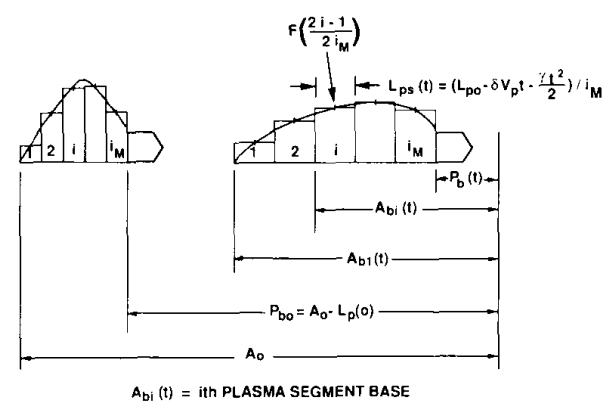

Fig. 2. Segmented plasma current distribution.

$i$ th segment (see Fig. 2). A one-dimensional form function will be defined to give the constant current of the $i$ th segment in terms of the midpoint of the $i$ th segment ( $(2 i$ $\left.-1) / 2 i_{m}\right) . i_{m}$ is the number of segments. The form function is defined such that it gives the fraction of the total current carried by the $i$ th segment, and the sum of the fractional currents must equal the total current (i.e., the sum of the form function over $i$ equals 1 , and the product of the form function and the total current is the $i$ th segment current). The current density for the $i$ th segment is given by the product of form function for the $i$ th segment and the total current divided by the area of the $i$ th segment as follows:

$$
\begin{aligned}
J_{i}(t) & =A_{\chi} F\left(\frac{2 i-1}{2 i_{m}}\right) I(t) / H_{p} L_{p i}(t) \\
A_{\chi} & \equiv 1 / \sum_{i=1}^{i_{m}} F\left(\frac{2 i-1}{2 i_{m}}\right), \quad i_{m}=\text { \# of segments. }
\end{aligned}
$$

The derivative of $\phi_{c i}(t)$ multiplied by the negative of the number of turns on the coil gives the coil voltage as a function of time,

$$
f_{i}(t)=-N d \phi_{c i}(t) / d t
$$

for the $i$ th element. The derivative of (3) may be taken after integration, or the derivative may be taken under the integral (the result is the same). The sum of the voltage for each element gives the total voltage on the coil as

$$
\begin{aligned}
f(t) & \equiv V_{p}(t)=\sum_{i=1}^{i_{m}} C_{l i}\left(I_{g 1 i}+I_{g 2 i}+I_{g 3 i}+I_{g 4 i}\right) \\
& \equiv \sum_{i=1}^{i_{m}} f_{i}(t) \\
C_{l i} & =-N \mu_{0} W A_{c} A_{\chi} F\left(\frac{2 i-1}{2 i_{m}}\right) / 4 \pi H_{p} \\
& =C_{I} A_{\chi} F\left(\frac{2 i-1}{2 i_{m}}\right), \quad A_{\chi}=1 / \sum_{i=1}^{i_{m}} F\left(\frac{2 i-1}{2 i_{m}}\right)
\end{aligned}
$$

where the segment current is $I_{i}(t)=A_{\chi} F\left((2 i-1) / 2 i_{m}\right)$ $I(t)$. 
The integrals are as follows:

$$
\begin{aligned}
I_{g 1 i}= & \frac{-I(t) A_{b i}^{\prime}(t)}{L_{p i}(t)}\left\{\left(1 / R_{p i}(t, 1)-1 / R_{0 i}(t, 1)\right)\right. \\
& \left.-\left(1 / R_{p i}(t, 0)-1 / R_{0 i}(t, 0)\right)\right\} \\
I_{g 2 i}= & \frac{I(t) L_{p i}^{\prime}(t)}{L_{p i}(t)}\left\{\frac{1}{R_{p i}(t, 1)}-\frac{1}{R_{0 i}(t, 1)}\right\} \\
I_{g 3 i}= & \frac{-I(t) L_{p i}^{\prime}(t)}{L_{p i}^{2}(t)}\left\{-\ln \left(\frac{R_{0 i}(t, 1)+L_{p i}(t)-A_{b i}(t)}{R_{p i}(t, 1)+L_{p i}(t)-A_{b i}(t)}\right)\right. \\
& \left.+\ln \left(\frac{R_{0 i}(t, 0)-A_{b i}(t)}{R_{p i}(t, 0)-A_{b i}(t)}\right\}\right) \\
I_{g 4 i}= & \frac{I^{\prime}(t)}{L_{p i}(t)}\left\{\ln \left[\frac{L_{i}(t, 1)+R_{0 i}(t, 1)}{L_{i}(t, 1)+R_{p i}(t, 1)}\right]\right. \\
& \left.-\ln \left[\frac{L_{i}(t, 0)+R_{0 i}(t, 0)}{L_{i}(t, 0)+R_{p i}(t, 0)}\right]\right\}
\end{aligned}
$$

where

$$
\begin{aligned}
R_{p i}(t, 1) & =\left[L_{i}^{2}(t, 1)+\left(H_{0}-H_{p}\right)^{2}\right]^{1 / 2}, \\
R_{0 i}(t, 1) & =\left[L_{i}^{2}(t, 1)+H_{0}^{2}\right]^{1 / 2}, \\
L_{i}(t, 1) & =A_{b i}(t)-L_{p i}(t), \\
R_{p i}(t, 0) & =\left[L_{i}^{2}(t, 0)+\left(H_{0}-H_{p}\right)^{2}\right]^{1 / 2}, \\
R_{0 i}(t, 0) & =\left[L_{i}^{2}(t, 0)+H_{0}^{2}\right]^{1 / 2}
\end{aligned}
$$

and

$$
L_{i}(t, 0)=A_{b i}(t) .
$$

The voltage function (7)-(11) is the same as given in [1], but has been simplified here.

If the plasma segments are defined as follows (refer to Fig. 2):

$$
A_{b i}(t)=A_{b 0 i}-\left[\left(V_{p}+\delta V_{p i}\right) t+\frac{\left(a_{p}+\delta a_{i}\right)}{2} t^{2}\right]
$$

where

$$
\begin{aligned}
A_{b 0 i}= & A_{b 0}-L_{p 0}(i-1) / i_{m}, \\
\delta V_{p i}= & \left(i_{m}-i+1\right) \delta V_{p} / i_{m}, \\
\delta a_{i}= & \left(i_{m}-i+1\right) \gamma / i_{m}, \\
A_{b i}(t)= & \text { position of the } i \text { th plasma segment } \\
& \text { base, } \\
V_{p}, a_{p}= & \text { projectile initial velocity } \\
& \text { and acceleration, } \\
V_{p}+\delta V_{p i}, a_{p}+\delta a_{i}= & \text { velocity and acceleration } \\
& \text { of segment base }
\end{aligned}
$$

then the segment boundaries are continuous, the segments bases may have accelerations and velocities different from the projectile, the total length of the plasma is independent of the number of segments, and the length of the segments is the same, independent of the segment number:

$$
\begin{array}{cl}
L_{p i}(t)=L_{p s}(t)=\left(L_{p 0}-\delta V_{p} t-\frac{\gamma t^{2}}{2}\right) / i_{m} \\
\text { segment length } \\
L_{p}(t)=L_{p s}(t) i_{m} \quad \text { plasma length. }
\end{array}
$$

With these definitions, the derivatives in the voltage function are

and

$$
A_{b i}^{\prime}(t)=-\left[V_{p}+\delta V_{p i}+\left(a_{p}+\delta a_{i}\right) t\right]
$$

$$
L_{p s}^{\prime}(t)=-\left[\delta V_{p}+\gamma t\right] / i_{m} .
$$

For the short time during the plasma passage, the current may be simulated as a linear function of time:

$$
\begin{aligned}
& I(t)=I_{0}+\beta t \\
& I^{\prime}(t)=\beta .
\end{aligned}
$$

The geometrical distribution of the plasma is described by the segment distribution function,

$$
A_{\chi} F\left(\frac{2 i-1}{2 i_{m}}\right) \text {. }
$$

For a Gaussian function, this is

$$
\begin{aligned}
A_{\chi} F\left[\chi_{i}\right] & =A_{\chi} \exp \left(-Z_{i}^{2} / 2\right) \\
Z_{i} & \equiv\left(\left(\chi_{i}-\bar{x}\right) / \sigma\right) \\
A_{\chi} & =1 / \sum_{i=1}^{i_{m}} \exp \left(-z_{i}^{2} / 2\right) \\
\chi_{i} & \equiv(2 i-1) / 2 i_{m}
\end{aligned}
$$

where $\bar{x}$ is the Gaussian mean, and $\sigma$ is the standard deviation, the parameters of the Gaussian distribution function.

A second form for the segment distribution function is the amplitude of the segment directly. In this case,

$$
F_{(x i)}=a_{i}, A_{\chi}=1 / \sum_{i=1}^{i_{m}} a_{i} .
$$

The amplitude and Gaussian form functions will be used in evaluating the test data.

It should be mentioned that the closed-form solution agrees with the numerical evaluation of (3) to four significant figures when five or more segments are used.

\section{Least-Square Fit to the Voltage Function}

The general form for the voltage function for a data time point $t_{k}$ is

$f\left(t_{k} ; p_{1}, p_{2}, \cdots p_{n p}\right)=\sum_{i=1}^{i_{m}} f_{i}\left(t_{k} ; i ; p_{1}, p_{2} \cdots p_{n p}\right)$ 
where $t_{k}=$ the data point time, and $p_{1}, p_{2}, \cdots p_{n p}=$ the function parameters.

The least-square equations for corrections to the parameters of the function compatible with the data points is

$$
\begin{aligned}
\sum_{i=1}^{N_{p}} A_{m l} \Delta p_{1} & =B_{m} \quad l=1,2 \cdots N_{p} \\
m & =1,2 \cdots N_{p} .
\end{aligned}
$$

The solution to this set of $N_{p}$ linear algebraic equations is the least-square solution for the parameter corrections where

$$
\begin{aligned}
A_{l m} & =\sum_{k=1}^{N} P_{1}\left(t_{k}\right) P_{m}\left(t_{k}\right), \\
B_{m} & =\sum_{K=1}^{N}\left(V_{k}-f\left(t_{k}\right)\right) P_{m}\left(t_{k}\right)
\end{aligned}
$$

and

$$
\begin{aligned}
P_{1}\left(t_{k}\right) & \equiv \partial f / \partial p_{1} ; P_{m} \equiv \partial f / \partial p_{m} \\
V_{k} & =\text { measured voltage at } t_{k}
\end{aligned}
$$

and $f\left(t_{k} ; p_{1}, p_{2} \cdots p_{n p}\right)$ is abbreviated by $f$ here and in some of the above equations.

The procedure outlined here applies to multiple as well as single probes. In fitting multiple probes, it is assumed that the fitted parameters (such as initial conditions, position, plasma length, velocity, plasma expansion rate, and form-function parameters) are the same for all the probes fitted. Of course, the different probes will have different constants associated with them, such as coil diameter, number of turns, and location. The algorithm accounts for this difference by flagging the specific probe points when evaluating the matrix and vector of (22). The plasma may be advanced in time using (12)-(17) along with the fitted parameters of the form function. This is one method used on the test data to evaluate the fitting procedure. The plasma parameters are fit to data from probes toward the breech, and this fit is advanced in time and compared with the fits of probes located closer to the muzzle.

The problem in solving (22) is in obtaining the partial derivatives of the voltage function. The parameters which should be fitted are the initial position of the base of the plasma $A_{b 0}$, the initial length of the plasma $L_{p 0}$, the expansion rate of the plasma $\delta V_{p}$, the velocity of the projectile $V_{p}$, and the parameters describing the geometrical distribution such as $\bar{x}$ and $\sigma$ for the Gaussian. Other parameters which may require fitting are the acceleration of the projectile $a_{p}$ and the accelerated expansion of the plasma $\gamma$. Parameters such as the area of the coil $A_{c}$ may be fitted to check on the validity of the model (the projectile velocity may also be measured by other sensors and also used as a check).

Obviously, the partials are very complicated. However, after a few are worked out, many of the others are repetitious. The partials which have been worked out, programmed, and debugged are most of those mentioned above (i.e., $A_{c}, A_{b 0}, L_{p 0}, V_{p}, \delta V_{p}$, the Gaussian function parameters $\bar{X}, \sigma$, and the amplitude function parameters $\left.a_{i}\right)$.

\section{Fitting Test Data}

In the following discussion the $\dot{B}$ data fitted was obtained from the firing of a $1-\mathrm{cm}$ square bore EM railgun. The parameters to be fitted are the area of coil (which can be converted to a diameter), the projectile velocity $V_{p}$, the initial plasma base position $A_{b 0}$, the initial length $L_{p 0}$, the expansion rate $\delta V$ of the plasma, and the form-function parameters. For the Gaussians, the parameters are $\bar{x}$ and $\sigma$, and for the amplitude functions the parameters are $a_{i}$ ( or $A_{\chi} a_{i}$, where $A_{\chi} a_{i}$ gives the fraction of the current carried by segment $i$ ).

An experiment was designed (see Fig. 3) to see if compatible current distribution fits could be obtained from probes of different sizes located at different distances from the bore, and on opposite sides of the bore. Simultaneous fits were made of probe sets located at different distances from the breech. An attempt was made (by using dowels as shown in Fig. 3) to accurately align the coils with their planes being perpendicular to the bore axis and with the coil center being midway between the rails. The probes were set up symmetrically at different stations along the bore. There were four stations, $5,6,7$, and 8 , located $25.4,30.48,35.54$, and $40.64 \mathrm{~cm}$ from the gun breech. Stations 5 and 6 had two symmetrically located outer coils of a $1.27-\mathrm{cm}$ diameter, and two inner coils of $0.635 \mathrm{~cm}$. The coils of any station were in the same plane. Stations 7 and 8 only had the inner coils.

The test shot was a normal-type shot from the PUG square bore gun [4]. The charge voltage was $500 \mathrm{~V}$, the projectile mass was $5.08 \mathrm{~g}$, and the injection velocity was approximately $500 \mathrm{~m} / \mathrm{s}$. The diagnostics on the test consisted of the breech and muzzle voltage, the total current from the integrated Rogowski coil measurement and the $B$-dot voltage signals taken at $0.5 \mu$ s intervals.

The first output of interest from the test was the remarkable similarity between the voltage on the outer coil on the right compared to the outer coil on the left. Fig. 4 shows a plot of the voltage signals from L5 and R5. 'The fact that these values are so close indicates that if there is filimentation in the main body of the plasma, the filaments are close together and symmetrically distributed across the bore.

Several multiple fits of the data were made. The probe voltages were used during the time period between 350 and $700 \mu \mathrm{s}$. During this time period the current did not change much and was about $195 \mathrm{KA}$. The acceleration of the projectile may be obtained from the fitting procedure; however, for this test the average velocity between consecutive probes was used to obtain an acceleration. Table I gives the average velocity between probes computed by dividing the distance between the probes $(5.08 \mathrm{~cm})$ by the

${ }^{1}$ L6 and R6 could not be compared since L6 had four turns and R6 had three turns. 


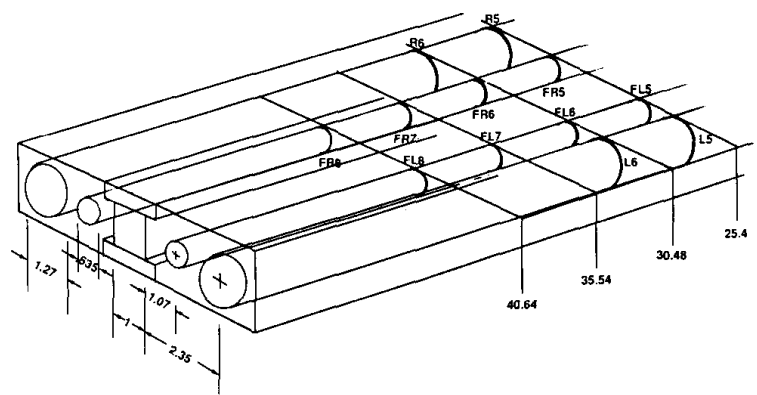

Fig. 3. Probe geometry for $B$-dot test.

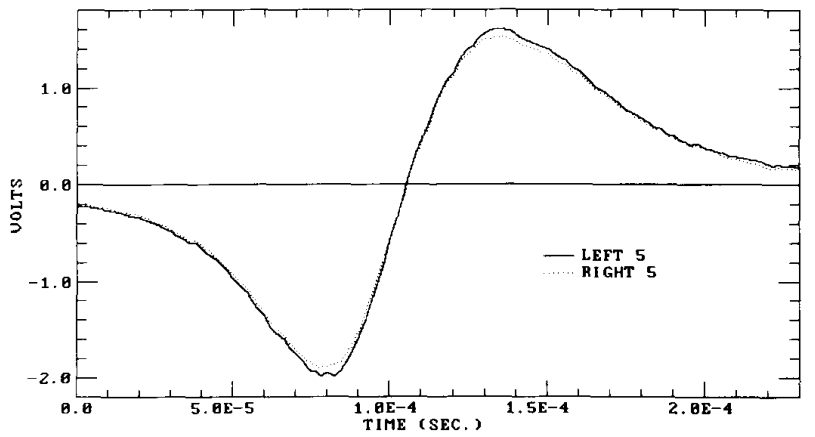

Fig. 4. B-dot voltage from symmetrically located probes.

TABLE I

average Velocity Between Probes

\begin{tabular}{l|c|c|c|c|c|c|c|c}
\hline \hline Probe $\rightarrow$ & L5-L6 & R5-R6 & FL5-FL6 & FR5-FR6 & FL6-FL7 & FR6-FR7 & FL7-FL8 & FR7-FR8 \\
\hline \hline $\begin{array}{l}\text { Station 5-6 } \\
\text { Station 6-7 } \\
\text { Station 7-8 }\end{array}$ & 832.8 & 832.8 & 832.8 & 829.4 & & & & \\
\hline
\end{tabular}

time difference between probe voltage maximum. The probes' first maximum corresponds approximately to the passage of the front face of the plasma (the proof of this was derived in [1] and [6]). The value used in the fitting for the acceleration was $700000 \mathrm{~m} / \mathrm{s}^{2}$. Since the measured diameter of the coil is not the effective diameter in terms of induced voltage, an effective diameter was obtained by fitting the data from each probe separately. The effective diameter was within 3 percent of the measured value.

The inputs for the fitting are the total current, the position, diameter, the number of turns on the coils, and the projectile acceleration. With this input, the time voltage data from sets of coils are fit simultaneously to determine the current distribution of the armature. Figs. 5, 6, and 7 are examples of two probe fits.

Fig. 5 shows the actual data plotted with the fitted data for probes L5-L6. The fitted data consists of the voltage function evaluated using the fitted parameters. Fig. 6 shows a plot of the current distribution taken from the fit given in Fig. 5. The fitted value of the initial plasma velocity and length, along with its expansion rate, is also given in Fig. 6. Fig. 7 is a fit of the opposing probes of Fig. 6 (i.e., R5-R6). Note that on probe R6, the voltage

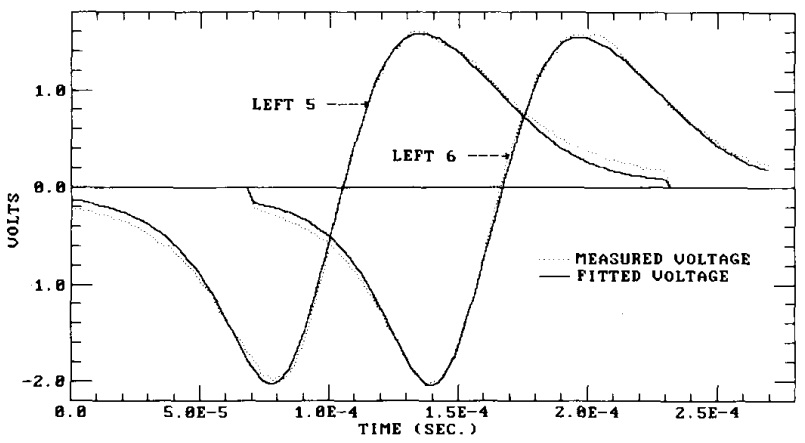

Fig. 5. Actual and fitted data for L5-L6 (amplitude 4 segments).

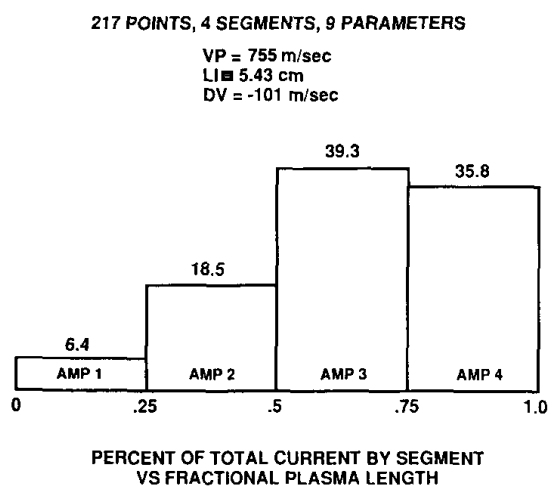

Fig. 6. Current distribution L5-L6 (amplitude 4 segments).

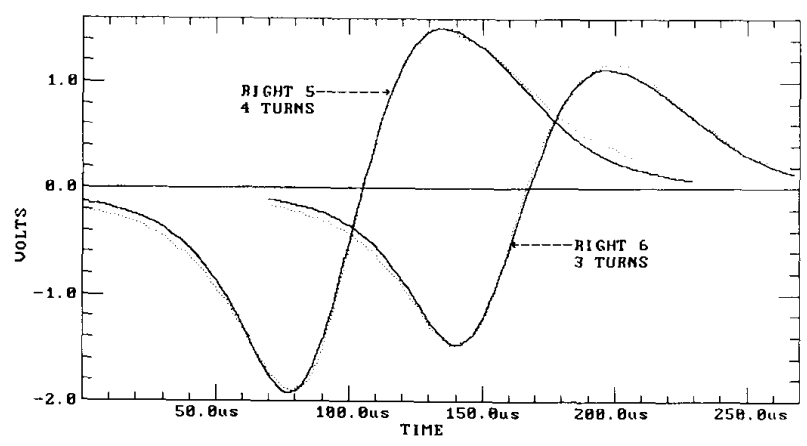

Fig. 7. Actual and fitted data for R5-R6 (amplitude 4 segments).

is lower at maximum by about $3 / 4$. This is because the probe was accidentally wound with three instead of four turns. Fig. 8 shows an example of a three-probe simultaneous fit. In this case, the amplitude form function was used and six segments were fit from the data of probes FR5, FR6, and FR7. The fit is designated FR5-FR6-FR7 (amplitude six segments).

Table II gives a series of fits for the probes used on the test. The table shows the probes which were fit simultaneously, the number of data points, and the fitted values for: The initial projectile velocity, the initial position of the plasma base, the initial length of the plasma, the contraction rate of the plasma (negative is expanding), the number of segments fit, and the fraction of the current carried by each segment. In the case of the Gaussian fit, 
TABLE II

LEAST-SQUare PARAMETER Fits

\begin{tabular}{|c|c|c|c|c|c|c|c|c|c|c|c|c|c|c|}
\hline \multirow[b]{2}{*}{ Probe Sets } & \multirow[b]{2}{*}{$\begin{array}{c}\# \\
\text { Pts. }\end{array}$} & \multirow[b]{2}{*}{ VP } & \multirow[b]{2}{*}{$A \phi$} & \multirow[b]{2}{*}{ LI } & \multirow[b]{2}{*}{ DV } & \multirow[b]{2}{*}{\begin{tabular}{|c|}
$\#$ \\
Segnents
\end{tabular}} & \multicolumn{6}{|c|}{ Segnent \% arrent } & \multirow[b]{2}{*}{$\overline{\bar{x}}$} & \multirow[b]{2}{*}{$\bar{\sigma}$} \\
\hline & & & & & & & 1 & 2 & 3 & 4 & 5 & 6 & & \\
\hline$L 5-16$ & 217 & 754.9 & .1172 & .054335 & -101.0 & 4 & 6.41 & 18.5 & 39.3 & 35.8 & & & & \\
\hline$R 5-R 6$ & 217 & 739.2 & .1173 & .055341 & -7.6 & 4 & 6.70 & 17.7 & 38.4 & 37.2 & & & & \\
\hline FR5 - FR6 & 194 & 739.0 & .1202 & .05680 & -23.4 & 4 & 7.02 & 19.4 & 34.9 & 38.6 & & & & \\
\hline FR5-FR6-FR7 & 292 & 738.7 & .1199 & .05672 & -29.6 & 4 & 5.96 & 19.49 & 35.9 & 38.7 & & & & \\
\hline FR5-FR6-FR7 & 292 & 740.1 & .1210 & .05843 & -30.4 & 6 & 3.20 & 7.29 & 15.8 & 22.15 & 28.1 & 23.5 & & \\
\hline $\begin{array}{l}\text { FR5-FR6-FR7 } \\
\text { Gaussian Fit }\end{array}$ & 292 & 739.4 & .1234 & .06053 & $-31.3 \mid$ & 6 & 2.38 & 6.69 & 14.8 & 22.95 & 27.96 & 25.76 & .784 & .315 \\
\hline FR7-FRB & 189 & 831.8 & .1467 & .06640 & +36.2 & 4 & 6.84 & 20.1 & 36.1 & 37.0 & & & & \\
\hline
\end{tabular}

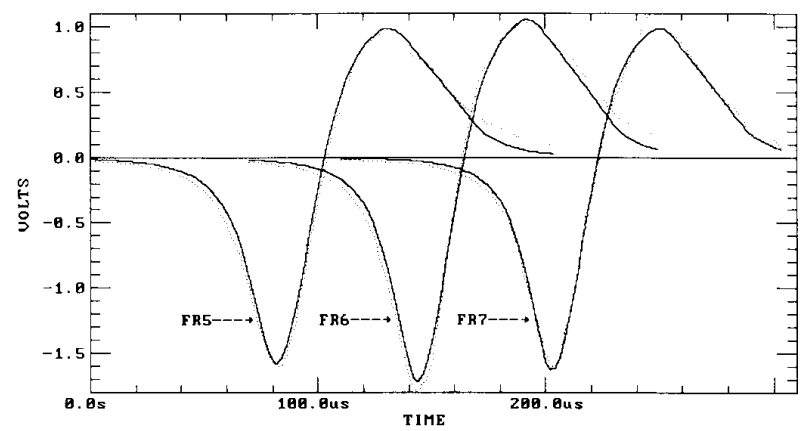

Fig. 8. Actual and fitted data for FR5-FR6-FR7 (amplitude 6 segments).

the fitted parameters are $\bar{x}$ and $\sigma$, and the percent current is calculated from these parameters.

The data reduction consisted of seven simultaneous fits. These fits are as follows:
L5-L6
R5-R6
FR5-FR6
(amplitude four segments), (amplitude four segments),
FR5-FR6-FR7 (Gaussian six segments),
FR7-FR8
(amplitude four segments).

Table III shows the comparison between some of the fitted parameters advanced in time and compared with other calculations of the same parameter, or compared to a fit made later in time. The first row of Table III gives the velocity midway between the probes calculated by dividing the distance between the probes by the time differ-
TABLE III

Comparison of Time-Advanced Fitted Parameter Between Fits and Other COMPUTATIONS

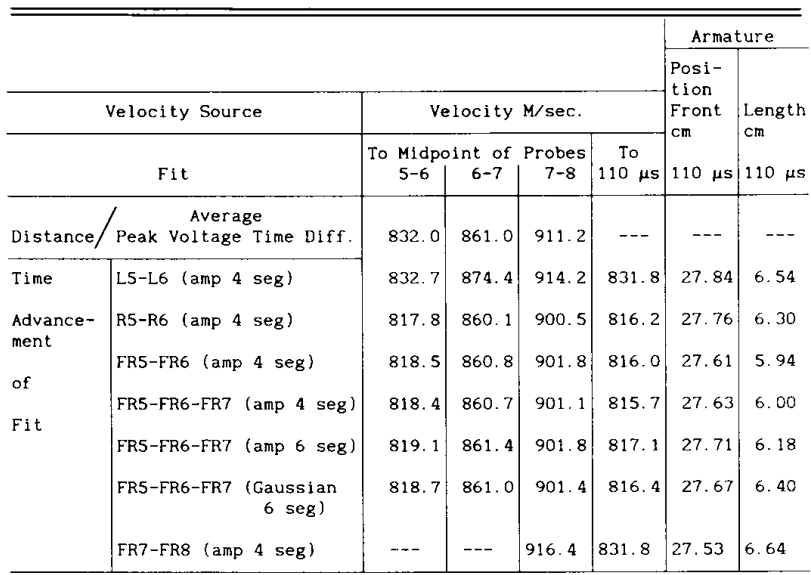


ence between the maximum voltage signal from both probes ([1] and [6] show that the maximum voltage corresponds approximately to the projectile base passage). The root mean squared difference between the probe-averaged velocity and the advanced-fitted velocity is between 8 and $10 \mathrm{~m} / \mathrm{s}$ for the seven fits, with L5-L6 giving the lower difference. The initial time on the fit FR7-FR8 (bottom row) was $110 \mu$ s later than the six other fits.

The last three columns give the velocity, plasma front position, and plasma length at $110 \mu \mathrm{s}$. For L7-L8, these are just the initial fitted values, but for all of the other fits these values are the initial parameter advanced in time. The velocity of L5-L6 compares well with FR7-FR8, and the rest of the fits differ by about $15 \mathrm{~m} / \mathrm{s}$. The maximum difference between the position of the plasma front is 3 $\mathrm{mm}$. The higher expansion rate of the outer-probe fits give a projected armature length between 1 and $2 \mathrm{~mm}$ of the FR7-FR8 fit. The expansion rate of the inner probes seems to be a little low. This may be due to the fact that the approximation made in (1) is not as valid for the inner as for the outer coils. The fit FR7-FR8 gives a small contraction rate and may indicate a change in the expansion rate at the later time, or may involve the approximation made in (1) as it effects the inner probes.

The data from probes FR5-FR6-FR7 was fitted in several different ways and then compared. These fits are an amplitude function four-segment fit, an amplitude function six-segment fit, and a Gaussian six-segment fit. The amplitude function six-segment fit is shown in Fig. 8. The initial current distributions for these fits are shown in Fig. 9. The percent of the total current carried by each segment is listed at the top of each segment. The forward half of the armature carries most of the current (about 76 percent for six-segment fits). The current density in the forward half ranges from 43 to $57 \mathrm{KA} / \mathrm{cm}^{2}$ for this shot where the current during data acquisition was $\sim 194 \mathrm{KA}$. All three fits give the same position of the front face of the plasma to within $0.6 \mathrm{~mm}$. All three distributions are nearly the same. The two six-segment fits are closer and probably represent the true current distribution better than the foursegment fit, since the four-segment fit was more constrained. The six-segment amplitude fit is less constrained than the Gaussian, but the Gaussian gives nearly the same distribution, which indicates that the closed-form Gaussian is acceptable for these operating conditions.

In order to correlate the $B$-dot current distribution analysis with other sensors, data from a 1986 test [6] using optical diagnostics was used. In this test a photo diode was directly opposite the $B$-dot coil located at station 7 ( $35.56 \mathrm{~cm}$ from the breech in a $60-\mathrm{cm}$ gun). A plot of the output of the two sensors is given in Fig. 10. Simple analysis assuming a uniform current distribution [1], [6] indicated that the front face of the current-carrying plasma is adjacent to the coil at the first voltage maxima. Fig. 10 shows the sharp rise in the photo-diode voltage (indicating a sharp rise in light intensity 350-1200 nm), about the time of $B$-dot maximum voltage. Actually, the photodiode rise occurs a little before the $B$-dot maximum (about $6 \mu \mathrm{s})$.

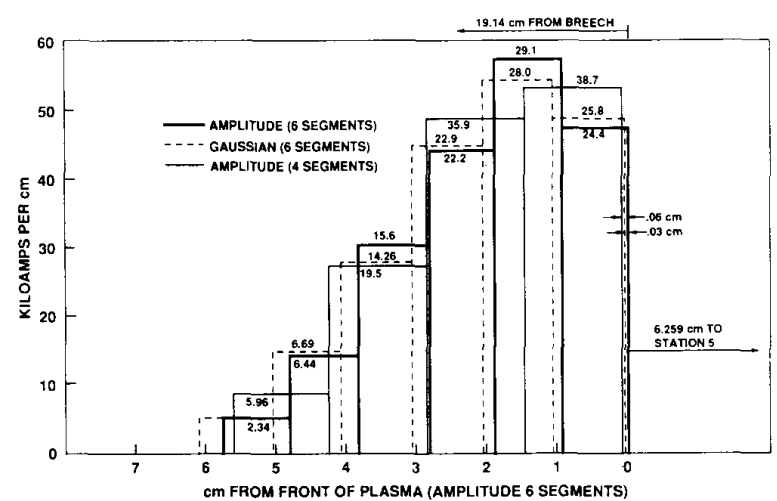

Fig. 9. Current distribution for FR5-FR6-FR7 fitted three ways.

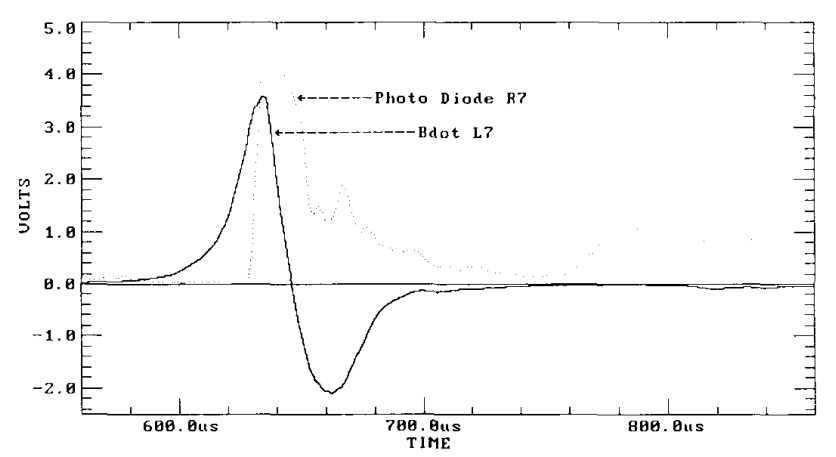

Fig. 10. Voltage output station 7.

The data from the $B$-dot station shown in Fig. 10, L7, and the $B$-dot station preceeding, L6, were fit simultaneously to give the fit shown in Fig. 11. The initial velocity on this fit was $1417 \mathrm{~m} / \mathrm{s}$, with the front face of the plasma being $6.43 \mathrm{~cm}$ from $B$-dot L6, giving a velocity at midpoint between L6 and L7 of $1567 \mathrm{~m} / \mathrm{s}$. The front face of the plasma, the velocity, and the plasma length may be advanced as previously done. The current distribution for this fit, L6-L7 (amplitude five segments), is shown in Fig. 12. The current distribution here is strung out more than the previous one. Eighty percent of the current is carried in the front $2 / 5$ of the plasma, with four percent carried in the last $2 / 5$.

Fig. 13 shows the progression of the front face of the armature on the same plot with the photo diode. The photo diode begins a sharp rise when the front face of the plasma (from $B$-dot analysis) is $1-\mathrm{cm}$ short of the photo-diode station. This can be accounted for in one of several ways: First, if the sharp rise in the photo diode corresponds to the passage of the front face of the plasma and the base of the projectile, then the projected position of the plasma front face is off by about $1 \mathrm{~cm}$; secondly, the projected position of the front face of the plasma is correct and also is at the back of the projectile, then the sharp rise in the photo diode occurs at the passage of the front face of the projectile (a $1-\mathrm{cm}$ cube). This would imply that there is a source of radiation sufficient to cause the rise between the projectile and the insulator; a third explanation would be that the front face of the plasma (ionized conducting gas) 


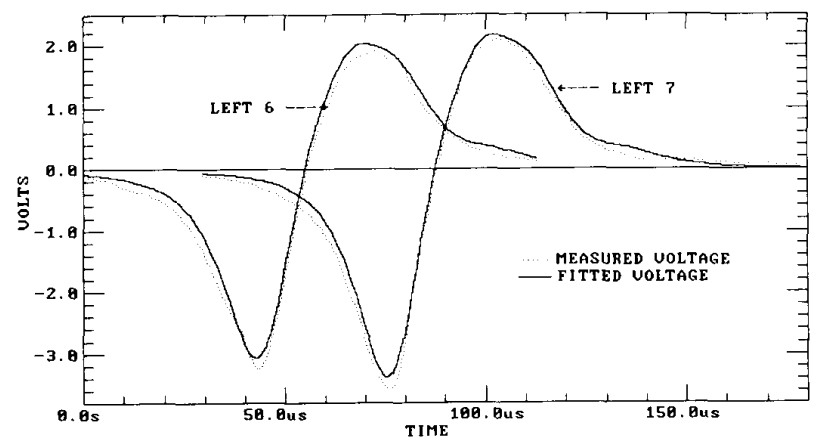

Fig, 11. Actual and fitted data L6-L7 (amplitude 5 segments), Aug. '86.

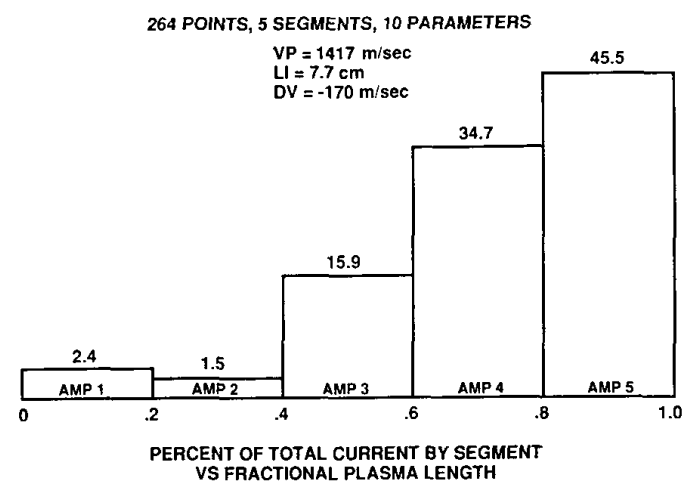

Fig. 12. Current distribution L6-L7 (amplitude 5 segments), Aug. ' 86.

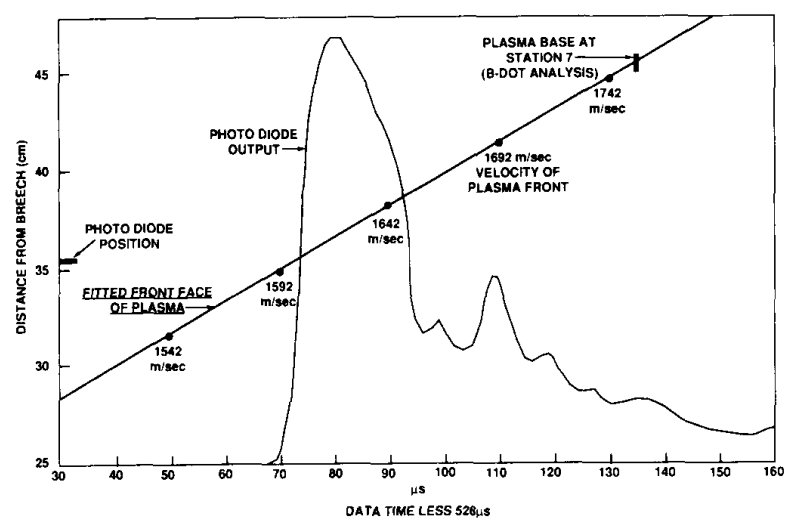

Fig. 13. Fitted position of projectile base with photo diode, Aug. '86. rent distribution in terms of its initial position and velocity, expansion rate, and geometrical variation in form in the bore directions. The algorithm developed was tested by comparing fits using different form functions, comparing fits using probes of different sizes, different positions along the bore, and at symmetrical positions on opposite sides of the bore. Fitted data was also compared to the data from a photo-diode sensor. The data fits using different form function gave essentially the same current distributions. The data indicated that fits from early probes could be moved forward in time, using the fitted parameters, and the advanced solution corresponded well to fits of probes located further down the bore. The voltage signature of two symmetrically placed outer probes, which were well aligned and wound exactly, gave essentially the same voltage signature. This may indicate no filamentation in the interior of the plasma or numerous uniform symmetrically distributed filaments. Comparison of the position of the front face of the plasma, as determined by the $B$-dot fit, with the photo-diode output leaves unanswered the exact (within $1 \mathrm{~cm}$ ) relative position of the front face of the plasma and the base of the projectile. These questions will be answered by further experiments.

Generally, the test indicates that the least-square method for determining current distribution gives consistent results and is compatible with other sensor data, is versatile by allowing several types of analysis, and is easily automated.

\section{REFERENCES}

[1] K. K. Cobb, "Determination of current distribution in EM gun armature by least squares fitting of $\dot{B}$ coil voltage," IEEE Trans. Plasma Sci., vol. 16, pp. 352-359, June 1988.

[2] K. A. Jamison, M. Marquez-Reines, and H. S. Burden, "Measurements of the spatial distribution of current in a railgun arc armature,' IEEE Trans. Magn., vol. Mag-20, pp. 403-406, Mar. 1984.

[3] J. D. Powell and J. H. Batteh, "Plasma dynamics of an arc-driven electromagnetic, projectile accelerator," J. Appl. Phys., vol. 52, pp. 2717-2730, Apr. 1981.

[4] J. D. Powell, "Plasma analysis of a large-bore, arc-driven railgun," presented at the 4th Symp. Electromagnetic Launch Tech., Austin, TX, Apr. 1988.

[5] D. Jensen, "Railgun configuration research at the Air Force Armament Laboratory," presented at the EM Gun Armature Workshop, Eglin AFB, FL, June 24-26, 1986.

[6] E. J. Clothiaux and K. K. Cobb, "Spectroscopic studies of a railgun arc," in Proc. 3rd Electromagnetic Launch Tech. Symp. (Austin, TX), Apr. 1986.

is $1-\mathrm{cm}$ behind the projectile base, and the nonconducting $1-\mathrm{cm}$ gas layer behind the projectile is warm enough to cause the sharp rise in the light intensity sensed by the photo diode.

\section{Conclusions}

The least-square procedure for the determination of the current distribution is a versatile and very useful tool for analyzing the characteristics of the railgun plasma armature. This method allows for the determination of the cur-

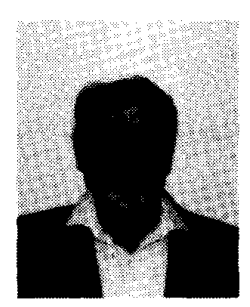

Kenneth K. Cobb was born in Milton, FL, on November 2, 1925. He received the B.S. (1955) and the M.S. (1957) degrees in physics from the University of Florida, Gainesville

Since 1959 he has worked for the Air Force as a Physicist in the development of conventional weapons. For the past six years he has been involved in the development of electromagnetic railguns. 